\title{
Feedback Stabilization for a Class of Nonlinear Stochastic Systems with State- and Control-Dependent Noise
}

\author{
Yu-Hong Wang, ${ }^{1}$ Tianliang Zhang, ${ }^{1}$ and Weihai Zhang ${ }^{2}$ \\ ${ }^{1}$ College of Information and Control Engineering, China University of Petroleum (East China), Qingdao 266580, China \\ ${ }^{2}$ College of Electrical Engineering and Automation, Shandong University of Science and Technology, Qingdao 266590, China \\ Correspondence should be addressed to Weihai Zhang; w_hzhang@163.com
}

Received 8 August 2014; Accepted 23 September 2014; Published 5 November 2014

Academic Editor: Ramachandran Raja

Copyright (c) 2014 Yu-Hong Wang et al. This is an open access article distributed under the Creative Commons Attribution License, which permits unrestricted use, distribution, and reproduction in any medium, provided the original work is properly cited.

\begin{abstract}
This paper mainly studies the state feedback stabilizability of a class of nonlinear stochastic systems with state- and controldependent noise. Some sufficient conditions on local and global state feedback stabilizations are given in linear matrix inequalities (LMIs) and generalized algebraic Riccati equations (GAREs). Some obtained results improve the previous work.
\end{abstract}

\section{Introduction}

Stability and stabilization are two important topics in modern control theory, which are first of considered issues in the systems analysis and synthesis. It is well known that stochastic control has become a very popular research area, which has been applied to mathematical finance [1], quantum systems [2], and so forth; stochastic stability and stabilization have been studied by many researchers; we refer the reader to the celebrated book [1] for the discussions of various stabilities. A series of works on robustly exponential stability can be found in [3-6]. While the pth moment stability were discussed in $[7,8]$, in particular, the asymptotic mean square stability has been studied for a long time; see [9-13]. The stabilizability of linear stochastic control systems has been investigated by $[9,10,12-17]$. In recent years, the study for stabilization of nonlinear stochastic systems has attracted great attention; the methods appearring in studying this topic can be summarized as follows: GARE-based method $[9,12,18,19]$; control Lyapunov function method $[1,3-6,20]$; passive system method [21], and spectral analysis method based on generalized Lyapunov operators $[13,16,17]$. We refer the reader to [19] for the stabilization of general nonlinear stochastic systems, where a class of new Hamilton-Jacobi inequalities were presented.

It can be seen that most of the previous works were on the systems with only the state-dependent noise.
In the present paper, we deal with a class of linearized systems with both the state- and control-dependent noise. Some sufficient conditions on local state feedback stabilization are given via LMIs and GAREs, respectively, which not only generalize but also improve the results of [18]. We also investigate the global state feedback stabilization and a sufficient condition is also given in terms of LMIs. A numerical example verifies the effectiveness of our results.

\section{Problem Setting}

Consider the following stochastic control system governed by Itô's differential equation:

$$
d x(t)=f(x(t), u(t)) d t+\sum_{i=1}^{l} \sigma_{i}(x(t), u(t)) d W_{i}(t) .
$$

In the above, $x(t) \in \mathscr{R}^{n}$ is called the system state and $u(t) \in \mathscr{R}^{m}$ the control input. $\left\{W_{i}(t), i=1, \ldots, l\right\}$ is the standard Wiener process defined on the probability space $(\Omega, \mathscr{F}, \mathscr{P})$ with a natural filter $\mathscr{F}_{t}=\sigma\left(W_{i}(s), 0 \leq s \leq\right.$ $t ; i=1, \ldots, l)$. Without loss of generality, we can suppose $\left\{W_{i}(t), i=1, \ldots, l\right\}$ are one-dimensional. Assume $u(t)$ is an adapted and measurable process with respect to $\mathscr{F}_{t}, f(0, u) \equiv$ $0, \sigma_{i}(0, u) \equiv 0$; that is, $x \equiv 0$ is an equilibrium point of (1). Under very general conditions on $f, \sigma_{i}(i=1, \ldots, l)$, and 
$u(t)$, stochastic control system (1) has a unique strong solution $x_{s, \xi}(t)$ for any $t \geq s \geq 0$ and the initial state $x(s)=\xi \in \mathscr{R}^{n}$; see $[1,22]$. We first introduce the following definition.

Definition 1. We say that the equilibrium point $x \equiv 0$ of system (1) is locally asymptotically stabilizable via a linear constant state feedback $u(t)=K x(t)$, if the solution $x \equiv 0$ of the closed-loop system,

$$
d x(t)=f(x(t), K x(t)) d t+\sum_{i=1}^{l} \sigma_{i}(x(t), K x(t)) d W_{i}(t)
$$

is asymptotically stable in probability [1]; that is, for any $s \geq 0$ and $\varepsilon>0$

$$
\begin{aligned}
& \lim _{\|\xi\| \rightarrow 0} \mathscr{P}\left(\sup _{t \geq s}\left\|x_{s, \xi}(t)\right\|>\varepsilon\right)=0, \\
& \lim _{\|\xi\| \rightarrow 0} \mathscr{P}\left(\lim _{t \rightarrow \infty} x_{s, \xi}(t)=0\right)=1,
\end{aligned}
$$

where $K$ is a constant matrix of suitable dimension. In addition, if the solution $x \equiv 0$ of the closed-loop system (2) is asymptotically stable in the large (see, e.g., [1]), that is, if both (3) and

$$
\mathscr{P}\left(\lim _{t \rightarrow \infty} x_{s, \xi}(t)=0\right)=1
$$

hold, then we say that the equilibrium point $x \equiv 0$ of system (1) is globally asymptotically stabilizable via a linear state feedback $u(t)=K x(t)$.

It is well known [1] that if there exists a neighborhood $U$ of the origin, a Lyapunov function $V(t, x) \in C_{2}^{0}(\{t>0\} \times U)$, $V(t, x)>0$ in domain $\{t>0\} \times U$, which has an infinitesimal upper limit, that is,

$$
\lim _{\|x\| \rightarrow 0} \sup _{t>0} V(t, x)=0
$$

satisfying

$$
\begin{aligned}
\mathscr{L} V(t, x)= & \frac{\partial V(t, x)}{\partial t}+f^{\prime}(x, K x) \frac{\partial V(t, x)}{\partial x} \\
& +\frac{1}{2} \sum_{i=1}^{l} \sigma_{i}^{\prime}(x(t), K x(t)) \\
& \times \frac{\partial^{2} V(t, x)}{\partial x^{2}} \sigma_{i}(x(t), K x(t))<0,
\end{aligned}
$$

then the solution $x(t) \equiv 0$ of system (2) is asymptotically stable in probability. If $V(t, x)$ also admits the following property,

$$
\inf _{t>0} V(t, x) \longrightarrow \infty, \quad \text { as }\|x\| \longrightarrow \infty
$$

then the solution $x(t) \equiv 0$ of system (2) is asymptotically stable in the large. $\mathscr{L}$ is the so-called infinitesimal generator of (2).

Now, suppose $f(x, u)$ and $\sigma_{i}(x, u)$ can be linearized as

$$
\begin{array}{cc}
f(x, u)=A x+B u+H_{0}(x, u), & H_{0}(0, u)=0, \\
\sigma_{i}(x, u)=C_{i} x+D_{i} u+H_{i}(x, u), & H_{i}(0, u)=0, \\
& i=1, \ldots, l,
\end{array}
$$

Respectively; then the linearized stochastic system of (1) is as

$$
\begin{aligned}
d x= & \left(A x+B u+H_{0}(x, u)\right) d t \\
& +\sum_{i=1}^{l}\left(C_{i} x+D_{i} u+H_{i}(x, u)\right) d W_{i},
\end{aligned}
$$

where $A, B, C_{i}, D_{i}, i=1, \ldots, l$, are constant matrices. In what follows, we will discuss the stabilization of (10).

\section{Locally Asymptotic Stabilization}

3.1. Main Results. In this section, we obtain two theorems on locally asymptotic stabilization of (10) as follows.

Theorem 2. Suppose

$$
\begin{array}{ll}
\lim _{\|x\| \rightarrow 0} \frac{\left\|H_{i}(x, K x)\right\|}{\|x\|}=0, & \forall K \in \mathscr{R}^{m \times n}, \\
& i=0,1, \ldots, l,
\end{array}
$$

and the following LMI,

$$
\left[\begin{array}{ccccc}
P A^{\prime}+A P+Y^{\prime} B^{\prime}+B Y & C_{1} P+D_{1} Y & C_{2} P+D_{2} Y & \cdots & C_{l} P+D_{l} Y \\
P C_{1}^{\prime}+Y^{\prime} D_{1}^{\prime} & -P & 0 & \cdots & 0 \\
P C_{2}^{\prime}+Y^{\prime} D_{2}^{\prime} & 0 & -P & \cdots & 0 \\
\vdots & \vdots & \vdots & \cdots & \vdots \\
P C_{l}^{\prime}+Y^{\prime} D_{l}^{\prime} & 0 & 0 & \cdots & -P
\end{array}\right]<0,
$$


has a solution $P>0, Y \in \mathscr{R}^{m \times n}$; then the equilibrium point $x \equiv 0$ of system (10) is locally asymptotically stabilizable in probability with control law

$$
u(t)=Y P^{-1} x(t) .
$$

The following theorem is another description for locally asymptotic stabilization in probability via GARE.

Theorem 3. Under the condition of (11), if for any $R>0, Q>$ 0, GARE,

$$
\begin{aligned}
P A & +A^{\prime} P+\sum_{i=1}^{l} C_{i}^{\prime} P C_{i}-\left(P B+\sum_{i=1}^{l} C_{i}^{\prime} P D_{i}\right) \\
& \times\left(R+\sum_{i=1}^{l} D_{i}^{\prime} P D_{i}\right)^{-1}\left(B^{\prime} P+\sum_{i=1}^{l} D_{i}^{\prime} P C_{i}\right)+Q=0,
\end{aligned}
$$

has a positive solution $P>0$, then system (10) is locally asymptotically stabilizable in probability with control law

$$
u(t)=-\left(R+\sum_{i=1}^{l} D_{i}^{\prime} P D_{i}\right)^{-1}\left(B^{\prime} P+\sum_{i=1}^{l} D_{i}^{\prime} P C_{i}\right) x(t) .
$$

To prove our main results, we first consider the linear constant coefficient stochastic control system

$$
d x=F x d t+\sum_{i=1}^{l} G_{i} x d W_{i}
$$

System (16) is said to be asymptotically mean square stable if, for any $x(s)=\xi, \lim _{t \rightarrow \infty} E\left\|x_{s, \xi}(t)\right\|^{2}=0$.

Lemma 4 (see [23]). System (16) is asymptotically mean square stable if and only if the following Lyapunov-type inequality,

$$
P F+F^{\prime} P+\sum_{i=1}^{l} G_{i}^{\prime} P G_{i}<0
$$

has at least one solution $P>0$.

Lemma 5 (see [13]). System (16) is asymptotically mean square stable if and only if its dual system,

$$
d x=F^{\prime} x d t+\sum_{i=1}^{l} G_{i}^{\prime} x d W_{i}
$$

is asymptotically mean square stable.

Proof of Theorem 2. By Schur's complement, LMI (12) is equivalent to the following inequality:

$$
\begin{aligned}
P A^{\prime}+ & A P+Y^{\prime} B^{\prime}+B Y \\
& +\sum_{i=1}^{l}\left(C_{i} P+D_{i} Y\right) P^{-1}\left(C_{i} P+D_{i} Y\right)^{\prime}<0,
\end{aligned}
$$

having a pair of solutions $P>0, Y \in \mathscr{R}^{m \times n}$. Let $Y=K P$; then (19) becomes

$$
\begin{aligned}
(A+ & B K) P+P(A+B K)^{\prime} \\
& +\sum_{i=1}^{l}\left(C_{i}+D_{i} K\right) P\left(C_{i}+D_{i} K\right)^{\prime}<0 .
\end{aligned}
$$

By Lemma 4, (20) implies

$$
d x=(A+B K)^{\prime} x d t+\sum_{i=1}^{l}\left(C_{i}+D_{i} K\right)^{\prime} x d W_{i}
$$

to be asymptotically mean square stable, which yields

$$
d x=(A+B K) x d t+\sum_{i=1}^{l}\left(C_{i}+D_{i} K\right) x d W_{i}
$$

to be also asymptotically mean square stable from Lemma 5. Again, by Lemma 4, there exists at least one solution $P_{1}>0$ satisfying

$$
\begin{aligned}
P_{1}(A & +B K)+(A+B K)^{\prime} P_{1} \\
& +\sum_{i=1}^{l}\left(C_{i}+D_{i} K\right)^{\prime} P_{1}\left(C_{i}+D_{i} K\right)<0 .
\end{aligned}
$$

Take the Lyapunov function $V(x)=x^{\prime} P_{1} x, u(t)=K x(t)=$ $Y P^{-1} x(t)$; then, for system (10),

$$
\begin{aligned}
& \mathscr{L} V(x)=\sum_{i=1}^{l}\left(C_{i} x+D_{i} K x+H_{i}(x, K x)\right)^{\prime} \\
& \times P_{1}\left(C_{i} x+D_{i} K x+H_{i}(x, K x)\right) \\
& +2\left\langle A x+B K x+H_{0}(x, K x), P_{1} x\right\rangle \\
& =x^{\prime}\left[P_{1}(A+B K)+(A+B K)^{\prime} P_{1}\right. \\
& \left.+\sum_{i=1}^{l}\left(C_{i}+D_{i} K\right)^{\prime} P_{1}\left(C_{i}+D_{i} K\right)\right] x \\
& +2 H_{0}^{\prime}(x, K x) P_{1} x \\
& +2 \sum_{i=1}^{l} H_{i}^{\prime}(x, K x) P_{1}\left(C_{i}+D_{i} K\right) x \\
& +\sum_{i=1}^{l} H_{i}^{\prime}(x, K x) P_{1} H_{i}(x, K x) .
\end{aligned}
$$

Let

$$
\begin{aligned}
-Q:= & P_{1}(A+B K)+(A+B K)^{\prime} P_{1} \\
& +\sum_{i=1}^{l}\left(C_{i}+D_{i} K\right)^{\prime} P_{1}\left(C_{i}+D_{i} K\right)
\end{aligned}
$$


then, by (23), $Q>0$. So

$$
\begin{gathered}
x^{\prime}\left[P_{1}(A+B K)+(A+B K)^{\prime} P_{1}\right. \\
\left.+\sum_{i=1}^{l}\left(C_{i}+D_{i} K\right)^{\prime} P_{1}\left(C_{i}+D_{i} K\right)\right] x \\
\quad=-x^{\prime} Q x \leq-\lambda_{\min }(Q)\|x\|^{2} .
\end{gathered}
$$

By the given condition (11), for any $\epsilon>0$, there exists $\delta>0$, such that when $\|x\|<\delta,\left\|H_{i}(x, K x)\right\| \leq \epsilon\|x\|, i=0,1, \ldots, l$. So

$$
\begin{aligned}
2 H_{0}^{\prime}( & x, K x) P_{1} x+2 \sum_{i=1}^{l} H_{i}^{\prime}(x, K x) P_{1}\left(C_{i}+D_{i} K\right) x \\
& +\sum_{i=1}^{l} H_{i}^{\prime}(x, K x) P_{1} H_{i}(x, K x) \\
\leq & \left(2 \epsilon+2 \epsilon \sum_{i=1}^{l}\left\|C_{i}+D_{i} K\right\|+l \epsilon^{2}\right) \\
& \times\left\|P_{1}\right\| \cdot\|x\|^{2} \leq \frac{1}{2} \lambda_{\min }(Q)\|x\|^{2} .
\end{aligned}
$$

If we take $\epsilon$ sufficiently small, such that

$$
2 \epsilon+2 \epsilon \sum_{i=1}^{l}\left\|C_{i}+D_{i} K\right\|+l \epsilon^{2} \leq \frac{\lambda_{\text {min }}(Q)}{2\left\|P_{1}\right\|},
$$

then (26) together with (27) gives

$$
\mathscr{L} V(x) \leq-\frac{1}{2} \lambda_{\min }(Q)\|x\|^{2}<0
$$

for $x \neq 0$. Therefore, the system (10) is locally asymptotically stabilizable in probability with control law

$$
u(t)=K x(t)=Y P^{-1} x(t) .
$$

The proof of Theorem 2 is completed.

Remark 6. If there is a constant matrix $K$ of suitable dimension such that system (22) is asymptotically mean square stable, then the following control system,

$$
d x=(A x+B u) d t+\sum_{i=1}^{l}\left(C_{i} x+D_{i} u\right) d W_{i}
$$

is called stabilizable in mean square sense $[9,12,13]$.

Proof of Theorem 3. Note that if we let

$$
K=-\left(R+\sum_{i=1}^{l} D_{i}^{\prime} P D_{i}\right)^{-1}\left(B^{\prime} P+\sum_{i=1}^{l} D_{i}^{\prime} P C_{i}\right),
$$

then GARE (14) can be written as

$$
\begin{aligned}
P(A & +B K)+(A+B K)^{\prime} P \\
& +\sum_{i=1}^{l}\left(C_{i}+D_{i} K\right)^{\prime} P\left(C_{i}+D_{i} K\right) \\
= & -Q-K^{\prime} R K<0 .
\end{aligned}
$$

By repeating the proof of Theorem 2, Theorem 3 is easy to be proved.

In fact, Theorems 2 and 3 are equivalent; this can be seen from the following proposition.

Proposition 7. If, for some $Q>0, R>0$, GARE (14) has a positive solution $P>0$, then LMI (12) is feasible with at least a pair of solutions $P_{1}>0, Y \in \mathscr{R}^{m \times n}$; conversely, if $L M I$ (12) has a pair of solutions $P_{1}>0, Y \in \mathscr{R}^{m \times n}$, then, for any $Q>0$, $R>0$, GARE (14) has a unique positive solution $P>0$.

Proof. If, for some $Q>0, R>0$, GARE (14) has a positive solution $P>0$, then from (33) together with Lemma 4, system (22) is asymptotically mean square stable. Accordingly, system (21) is also asymptotically mean square stable by Lemma 5 . Again, by Lemma 4, there exists $P_{1}>0$, such that

$$
\begin{aligned}
P_{1}(A & +B K)^{\prime}+(A+B K) P_{1} \\
& +\sum_{i=1}^{l}\left(C_{i}+D_{i} K\right) P_{1}^{-1}\left(C_{i}+D_{i} K\right)^{\prime}<0 .
\end{aligned}
$$

Let $Y=K P_{1}$; then (34) follows

$$
\begin{aligned}
P_{1} A^{\prime} & +A P_{1}+Y^{\prime} B^{\prime}+B Y \\
& +\sum_{i=1}^{l}\left(C_{i} P_{1}+D_{i} Y\right) P_{1}^{-1}\left(C_{i} P_{1}+D_{i} Y\right)^{\prime}<0 .
\end{aligned}
$$

By Schur's complement, $P_{1}>0$ and $Y$ are also the solutions of (12). Conversely, if (12) has a pair of solutions $P_{1}>0, Y \in$ $\mathscr{R}^{m \times n}$, then, from the same discussion as above, system (22) is mean square stable. So (31) is stabilizable in mean square sense. From $[9,13]$, for any $Q>0, R>0$, GARE (14) has a unique positive solution $P>0$.

Remark 8. Although Theorem 2 is equivalent to Theorem 3, it seems that Theorem 2 is more convenient in actual use than Theorem 3, because we can easily test whether or not LMI (12) is feasible by existing convex optimization tools; see [10, 24]. However, we would like to point out that if GARE (14) has a positive solution $P>0$, by applying Theorem 10 of [9], $P$ must solve the following semidefinite programming problem:

$$
\max \operatorname{Tr}(P),
$$


subject to

$$
\left[\begin{array}{cc}
P A+A^{\prime} P+\sum_{i=1}^{l} C_{i}^{\prime} P C_{i}+Q & P B+\sum_{i=1}^{l} C_{i}^{\prime} P D_{i} \\
B^{\prime} P+\sum_{i=1}^{l} D_{i}^{\prime} P C_{i} & R+\sum_{i=1}^{l} D_{i}^{\prime} P D_{i}
\end{array}\right] \leq 0,
$$

The semidefinite programming problem (36)-(37), as LMI (12), can also be verified via some convex optimization tools $[10,24]$.

3.2. Comparison with the Existing Results. In (10), if we let $D_{i}=0, H_{i}(x, u) \equiv 0$ for $i=1, \ldots, l$, then the linearized system of (1) becomes

$$
d x=\left(A x+B u+H_{0}(x, u)\right) d t+\sum_{i=1}^{l} C_{i} x d W_{i} .
$$

By means of GARE-based method, the following result was obtained in [18].

Theorem 9. If for any real matrix $M \geq 0$, there exists a constant $\lambda>0$, such that

$$
\sum_{i=1}^{l} C_{i}^{\prime} M C_{i} \leq \lambda M
$$

Moreover, suppose $\lim _{\|x\| \rightarrow 0}\left(\left\|H_{0}(x, K x)\right\| /\|x\|\right)=0, \forall K \in$ $\mathscr{R}^{m \times n} ;(A, B)$ is controllable; $\left(Q^{1 / 2}, A\right)$ is observable with $Q \geq$ 0 ; then system (38) is locally asymptotically stabilizable in probability with the control law

$$
u(t)=-\frac{1}{2} R^{-1} B^{\prime} P x(t), \quad R>0,
$$

where $P>0$ is a unique solution of GARE

$$
P A+A^{\prime} P+\sum_{i=1}^{l} C_{i}^{\prime} P C_{i}-P B R^{-1} B^{\prime} P+Q=0 .
$$

Based on Theorem 9, we give the following remarks.

Remark 10. It is not convenient to use Theorem 9 in practice, because the condition (39) is difficult to verify for all real nonnegative symmetric matrices.

Remark 11. Checking the proof of Theorem 9 in [18], we can find that Theorem 9 of [18] required that the smallest eigenvalue of $Q$ should be larger than zero; that is, $Q>0$; so $\left(Q^{1 / 2}, A\right)$ is certainly observable.
GARE (41) is a special case of (14). We should point out that (39) and the controllability of $(A, B)$ are only sufficient but not necessary conditions for the existence of positive solutions of GARE (41) with $Q>0, R>0$; see [25] and the following counterexample.

Example 12. In GARE (41), we set $l=1, R=1, Q=I$, and

$$
\begin{aligned}
A & =\left[\begin{array}{cc}
0 & 1 \\
0 & -1
\end{array}\right], \\
B & =\left[\begin{array}{c}
0 \\
-1
\end{array}\right], \\
C_{1} & =\left[\begin{array}{ll}
0 & 0 \\
n & n
\end{array}\right], \quad n>0 .
\end{aligned}
$$

In this case, GARE (41) reduces to

$$
P A+A^{\prime} P+C_{1}^{\prime} P C_{1}-P B B^{\prime} P+I=0 .
$$

It is easy to test that

$$
d x=(A x+B u) d t+C_{1} x d W_{1}
$$

is stabilizable in mean square sense. By $[9,13],(43)$ must have a unique positive definite solution $P>0$. However, (39) is not satisfied; this can be seen by setting

$$
M=\left[\begin{array}{cc}
0 & 0 \\
0 & m_{22}
\end{array}\right], \quad m_{22}>0 .
$$

Considering Proposition 7, Theorem 2 not only has computational advantage but also generalizes and improves Theorem 9 given in [18].

Remark 13. In general, feedback stabilizing control laws are not unique; for example, in Theorem 9, except for $u(t)=$ $-(1 / 2) R^{-1} B^{\prime} P x(t), u(t)=-R^{-1} B^{\prime} P x(t)$ is another locally feedback stabilizing control law of system (38).

\section{Globally Asymptotic Stabilization}

Theorem 14. Suppose there exists a scalar $\lambda>0$, such that, for any $K \in \mathscr{R}^{m \times n}$ and $x \in \mathscr{R}^{n}$,

$$
H_{i}(x, K x) H_{i}^{\prime}(x, K x) \leq \lambda x x^{\prime}, \quad i=0,1, \ldots, l,
$$

and the following LMI, 


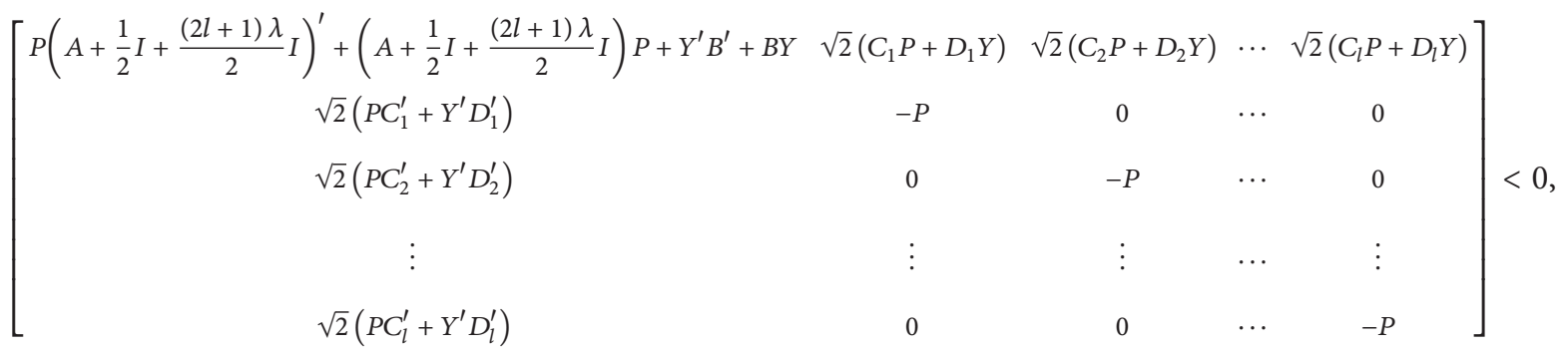

has solutions $P>0, Y \in \mathscr{R}^{m \times n}$; then the equilibrium point $x \equiv 0$ of system (10) is globally asymptotically stabilizable with the control law

$$
u(t)=Y P^{-1} x(t)
$$

Proof. Similar to the proof of Theorem 2, by Schur's complement, (47) is equivalent to the fact that there exist $P>0, Y \in$ $\mathscr{R}^{m \times n}$, such that

$$
\begin{aligned}
P(A & \left.+\frac{1}{2} I+\frac{(2 l+1) \lambda}{2} I\right)^{\prime} \\
& +\left(A+\frac{1}{2} I+\frac{(2 l+1) \lambda}{2} I\right) P+Y^{\prime} B^{\prime}+B Y \\
& +2 \sum_{i=1}^{l}\left(C_{i} P+D_{i} Y\right) P^{-1}\left(C_{i} P+D_{i} Y\right)^{\prime}<0 .
\end{aligned}
$$

Let $Y=K P$; then (49) implies that there exists a solution $P_{1}>0$ to

$$
\begin{aligned}
P_{1}(A & \left.+B K+\frac{1}{2} I+\frac{(2 l+1) \lambda}{2} I\right) \\
& +\left(A+B K+\frac{1}{2} I+\frac{(2 l+1) \lambda}{2} I\right)^{\prime} P_{1} \\
& +2 \sum_{i=1}^{l}\left(C_{i}+D_{i} K\right)^{\prime} P_{1}\left(C_{i}+D_{i} K\right)<0 .
\end{aligned}
$$

Still take the Lyapunov function $V(x)=x^{\prime} P_{1} x, u(t)=$ $K x(t)=Y P^{-1} x(t)$; then $V(x)$ satisfies (8) and (24). It is well known that

$$
2 H_{0}^{\prime}(x, K x) P_{1} x \leq H_{0}^{\prime}(x, K x) P_{1} H_{0}(x, K x)+x^{\prime} P_{1} x
$$

By (46), it concludes

$$
H_{0}^{\prime}(x, K x) P_{1} H_{0}(x, K x) \leq \lambda x^{\prime} P_{1} x
$$

So

$$
2 H_{0}^{\prime}(x, K x) P_{1} x \leq(\lambda+1) x^{\prime} P_{1} x .
$$

Similarly,

$$
\begin{aligned}
2 \sum_{i=1}^{l} H_{i}^{\prime}(x, K x) P_{1}\left(C_{i}+D_{i} K\right) x \\
\leq \sum_{i=1}^{l} H_{i}^{\prime}(x, K x) P_{1} H_{i}(x, K x) \\
\quad+\sum_{i=1}^{l} x^{\prime}\left(C_{i}+D_{i} K\right)^{\prime} P_{1}\left(C_{i}+D_{i} K\right) x \\
\leq(\lambda l) x^{\prime} P_{1} x+\sum_{i=1}^{l} x^{\prime}\left(C_{i}+D_{i} K\right)^{\prime} P_{1}\left(C_{i}+D_{i} K\right) x, \\
\quad \sum_{i=1}^{l} H_{i}^{\prime}(x, K x) P_{1} H_{i}(x, K x) \leq(\lambda l) x^{\prime} P_{1} x .
\end{aligned}
$$

Repeating the same procedure as in Theorem 2, we can prove $\mathscr{L} V(x)<0$ for all $x \in \mathscr{R}^{n}$. The theorem is shown.

Remark 15. Obviously, (11) and (46) do not imply each other, which motivates us to search for other less conservative conditions in the future.

\section{Numerical Example}

In this section, we present the following numerical example to illustrate the effectiveness of our main results.

Example 1. Consider the following two-dimensional nonlinear stochastic system:

$$
\begin{aligned}
d x= & \left(A x+B u+H_{0}(x, u)\right) d t \\
& +\left(C_{1} x+D_{1} u+H_{1}(x, u)\right) d W_{1}
\end{aligned}
$$

with

$$
\begin{array}{cc}
A=\left[\begin{array}{cc}
-1 & 2 \\
0.5 & 1
\end{array}\right], \quad B=\left[\begin{array}{l}
0 \\
1
\end{array}\right], \\
C_{1}=\left[\begin{array}{cc}
2 & 0.5 \\
0 & 1
\end{array}\right], \quad D_{1}=\left[\begin{array}{l}
1 \\
0
\end{array}\right], \\
H_{0}(x, u)=2 x u, \quad H_{1}(x, u)=-x u .
\end{array}
$$




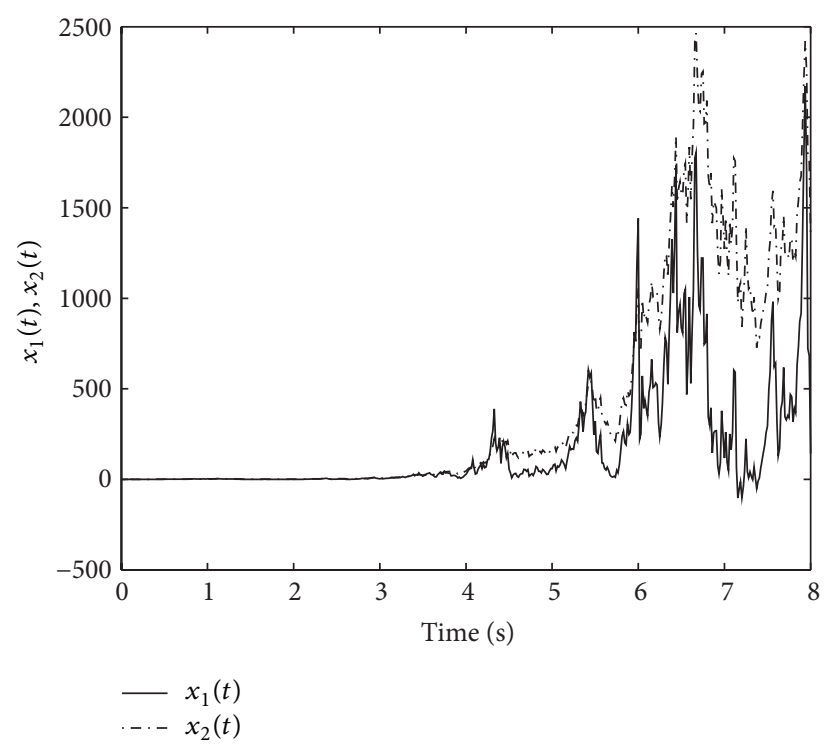

FIGURE 1: The state responses of the unforced system $(u=0)$.

Obviously, $H_{0}(x, u)$ and $H_{1}(x, u)$ satisfy condition (11). According to Theorem 2, a feasible solution is derived by solving LMI (12):

$$
\begin{gathered}
P=\left[\begin{array}{ll}
5.1894 & 0.4095 \\
0.4095 & 3.8125
\end{array}\right], \\
Y=\left[\begin{array}{ll}
-6.7947 & -6.5819
\end{array}\right] .
\end{gathered}
$$

Therefore, the control gain matrix is

$$
K=Y P^{-1}=\left[\begin{array}{ll}
-1.1831 & -1.5993
\end{array}\right] .
$$

The state responses of the unforced system $(u=0)$ and the controlled system $(u=K x)$ are shown in Figures 1 and 2, respectively. From Figure 2, it can be found that the controlled system can achieve stability by using the proposed controller.

\section{Conclusion}

In this paper, we have studied the feedback stabilizability of nonlinear stochastic systems with state- and controldependent noise. Some sufficient conditions on stabilization have been derived in terms of LMIs and GAREs. A numerical example is presented to show the validity of the obtained results.

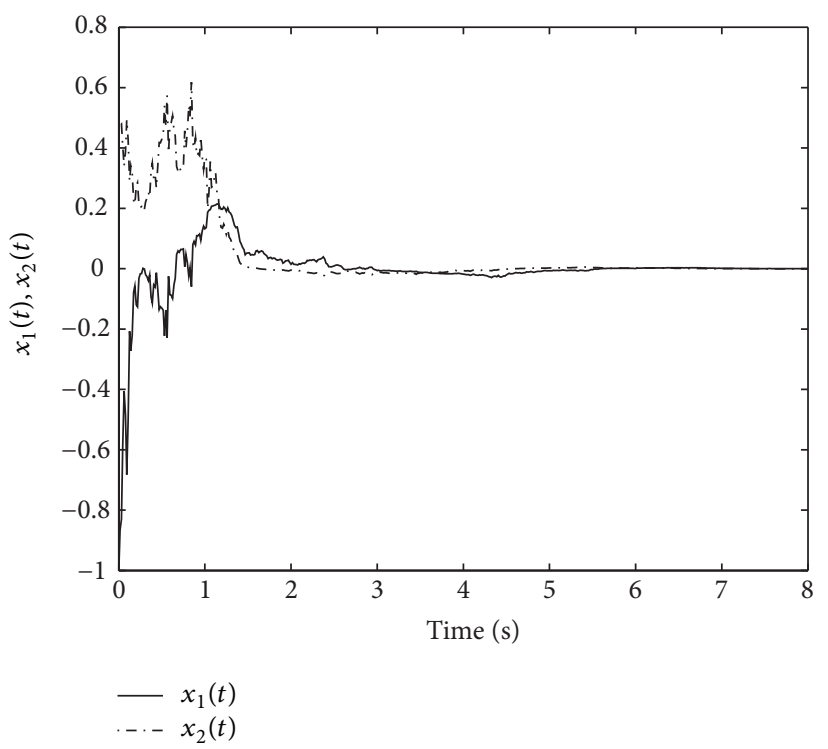

FIGURE 2: The state responses of the controlled system $(u=K x)$.

\section{Notations}

$\begin{array}{ll}\mathcal{S}_{n}: & \begin{array}{l}\text { The set of all } n \times n \text { symmetric } \\ \text { matrices }\end{array} \\ A^{\prime}: & \text { Transpose of a matrix } A \\ A \geq 0(A>0): & \begin{array}{l}\text { Positive semidefinite (positive } \\ \text { definite) symmetric matrix } A\end{array} \\ I: & \text { Identity matrix } \\ \operatorname{Tr}(P): & \text { Trace of a square matrix } P \\ C_{2}^{0}(\{t>0\} \times U): & \text { Class of functions } V(t, x) \text { twice } \\ & \text { continuously differential with respect } \\ & \text { to } x \in U \text { and once continuously } \\ & \text { differential with respect to } t>0 \\ & \text { except possibly at the point } x=0 .\end{array}$

\section{Conflict of Interests}

The authors declare that there is no conflict of interests regarding the publication of this paper.

\section{Acknowledgments}

This work is supported by the National Natural Science Foundation of China (no. 61174078), the Research Fund for the Taishan Scholar Project of Shandong Province of China and SDUST Research Fund (no. 2011KYTD105), and State Key Laboratory of Alternate Electrical Power System with Renewable Energy Sources (Grant no. LAPS13018).

\section{References}

[1] X. Mao, Stochastic Differential Equations and Their Applications, Horwood Publishing Series in Mathematics \& Application, Horwood Publishing, Chichester, UK, 1997.

[2] W. Zhang and B.-S. Chen, "Stochastic affine quadratic regulator with applications to tracking control of quantum systems," Automatica, vol. 44, no. 11, pp. 2869-2875, 2008. 
[3] Q. Zhu and J. Cao, "Stability analysis of markovian jump stochastic BAM neural networks with impulse control and mixed time delays," IEEE Transactions on Neural Networks and Learning Systems, vol. 23, no. 3, pp. 467-479, 2012.

[4] Q. Zhu, F. Xi, and X. Li, "Robust exponential stability of stochastically nonlinear jump systems with mixed time delays," Journal of Optimization Theory and Applications, vol. 154, no. 1, pp. 154-174, 2012.

[5] Q. Zhu and J. Cao, "Exponential stability of stochastic neural networks with both Markovian jump parameters and mixed time delays," IEEE Transactions on Systems, Man, and Cybernetics B: Cybernetics, vol. 41, no. 2, pp. 341-353, 2011.

[6] Q. Zhu and J. Cao, "Robust exponential stability of markovian jump impulsive stochastic Cohen-Grossberg neural networks with mixed time delays," IEEE Transactions on Neural Networks, vol. 21, no. 8, pp. 1314-1325, 2010.

[7] Q. Zhu, "Asymptotic stability in the pth moment for stochastic differential equations with Lévy noise," Journal of Mathematical Analysis and Applications, vol. 416, no. 1, pp. 126-142, 2014.

[8] Q. Zhu, "pth moment exponential stability of impulsive stochastic functional differential equations with Markovian switching," Journal of the Franklin Institute, vol. 351, no. 7, pp. 3965-3986, 2014.

[9] M. A. Rami and X. Y. Zhou, "Linear matrix inequalities, Riccati equations, and indefinite stochastic linear quadratic controls," IEEE Transactions on Automatic Control, vol. 45, no. 6, pp. 1131$1143,2000$.

[10] S. Boyd, L. El Ghaoui, E. Feron, and V. Balakrishnan, Linear Matrix Inequalities in System and Control Theory, vol. 15 of SIAM Studies in Applied Mathematics, Society for Industrial and Applied Mathematics (SIAM), Philadelphia, Pa, USA, 1994.

[11] A. Haghighi and S. M. Hosseini, "Analysis of asymptotic meansquare stability of a class of Runge-Kutta schemes for linear systems of stochastic differential equations," Mathematics and Computers in Simulation, vol. 105, pp. 17-48, 2014.

[12] J. L. Willems and J. C. Willems, "Feedback stabilizability for stochastic systems with state and control dependent noise," Automatica, vol. 12, no. 3, pp. 277-283, 1976.

[13] W. Zhang and B.-S. Chen, "On stabilizability and exact observability of stochastic systems with their applications," Automatica, vol. 40, no. 1, pp. 87-94, 2004.

[14] E. K. Boukas, "Stabilization of stochastic singular nonlinear hybrid systems," Nonlinear Analysis: Theory, Methods \& Applications, vol. 64, no. 2, pp. 217-228, 2006.

[15] S. Sathananthan, C. Beane, G. S. Ladde, and L. H. Keel, "Stabilization of stochastic systems under Markovian switching," Nonlinear Analysis. Hybrid Systems, vol. 4, no. 4, pp. 804-817, 2010.

[16] W. Zhang and B.-S. Chen, " $\mathscr{H}$-representation and applications to generalized Lyapunov equations and linear stochastic systems," IEEE Transactions on Automatic Control, vol. 57, no. 12, pp. 3009-3022, 2012.

[17] W. Zhang and L. Xie, "Interval stability and stabilization of linear stochastic systems," IEEE Transactions on Automatic Control, vol. 54, no. 4, pp. 810-815, 2009.

[18] Z. Y. Gao and N. U. Ahmed, "Feedback stabilizability of nonlinear stochastic systems with state-dependent noise," International Journal of Control, vol. 45, no. 2, pp. 729-737, 1987.

[19] W. Zhang, B.-S. Chen, and Z. Yan, "Feedback stabilization for nonlinear affine stochastic systems," International Journal of Innovative Computing, Information and Control, vol. 7, no. 9, pp. 5363-5375, 2011.
[20] P. Florchinger, "Feedback stabilization of affine in the control stochastic differential systems by the control Lyapunov function method," SIAM Journal on Control and Optimization, vol. 35, no. 2, pp. 500-511, 1997.

[21] P. Florchinger, "A passive system approach to feedback stabilization of nonlinear control stochastic systems," SIAM Journal on Control and Optimization, vol. 37, no. 6, pp. 1848-1864, 1999.

[22] B. Øksendal, Stochastic Differential Equations: An Introduction with Applications, Springer, New York, NY, USA, 6th edition, 2010.

[23] G. Da Prato and J. Zabczyk, Stochastic Equations in Infinite Dimensions, vol. 44 of Encyclopedia of Mathematics and its Applications, Cambridge University Press, Cambridge, UK, 1992.

[24] P. Gahinet, A. Nemirovski, A. J. Laub, and M. Chilali, LMI Control Toolbox, Math Works, Natick, Mass, USA, 1995.

[25] W. Zhang, "A study on positive solutions of generalized algebraic Riccati equation," Acta Automatica Sinica, vol. 27, no. 1, pp. 125-130, 2001 (Chinese). 


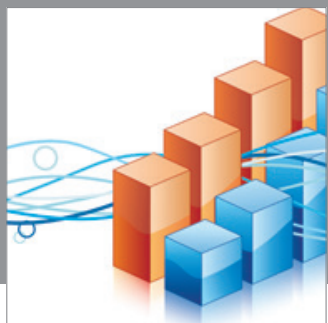

Advances in

Operations Research

mansans

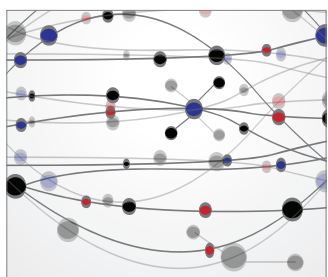

The Scientific World Journal
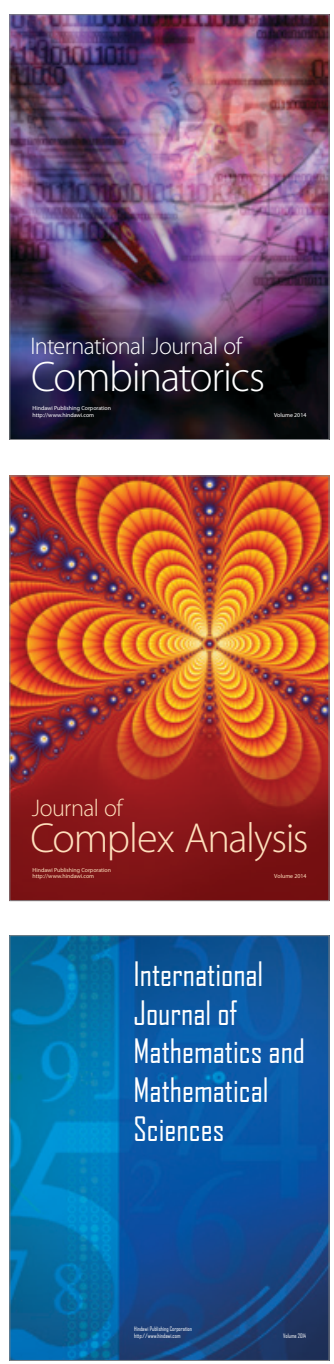
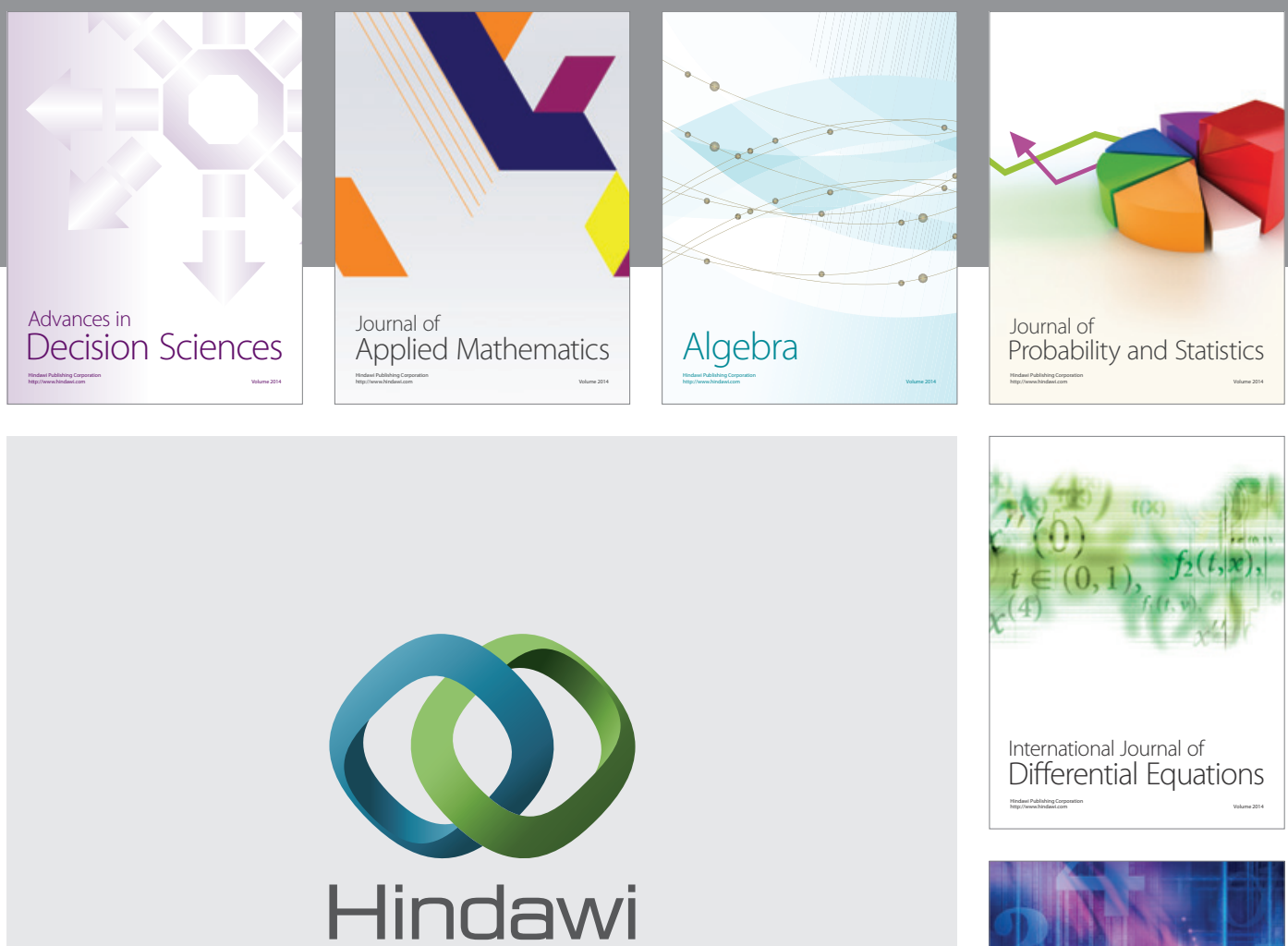

Submit your manuscripts at http://www.hindawi.com
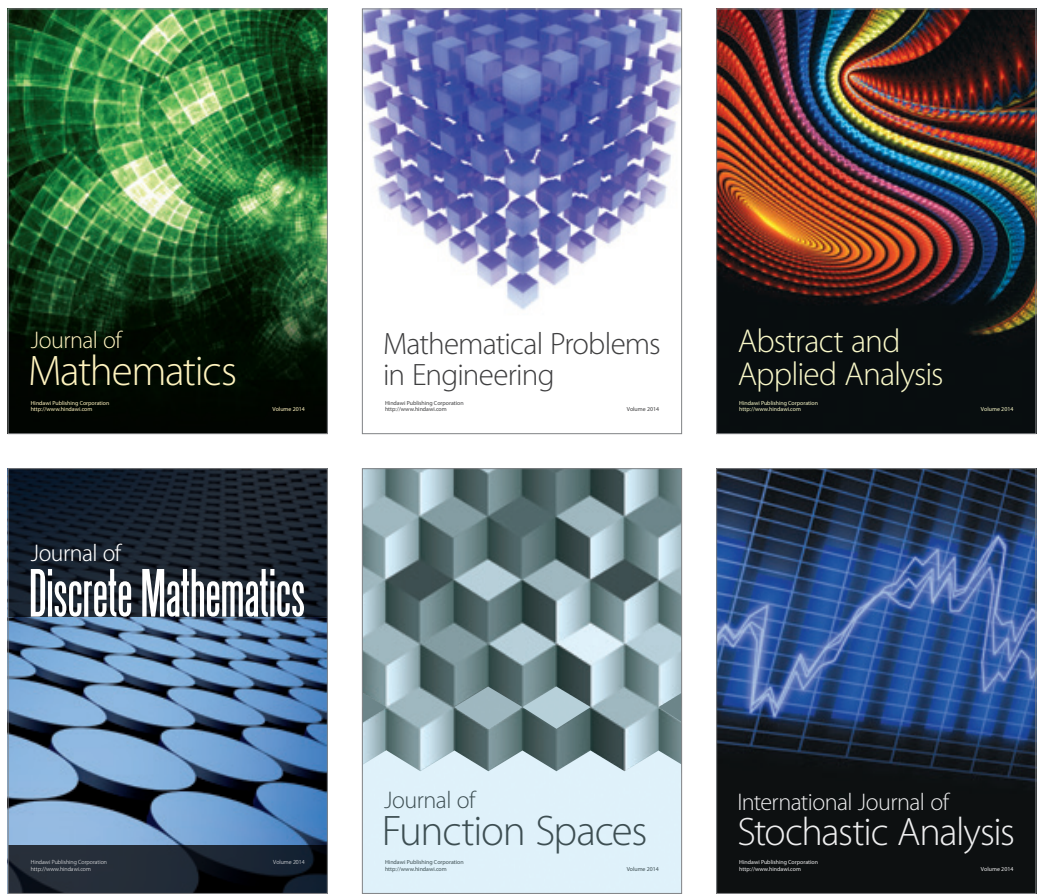

Journal of

Function Spaces

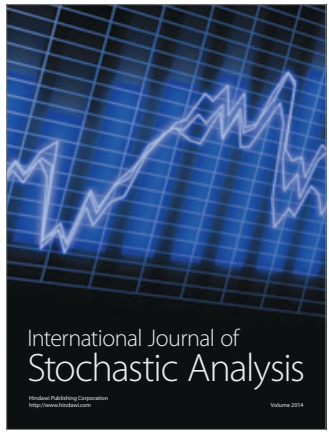

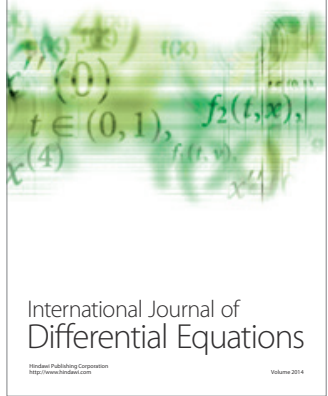
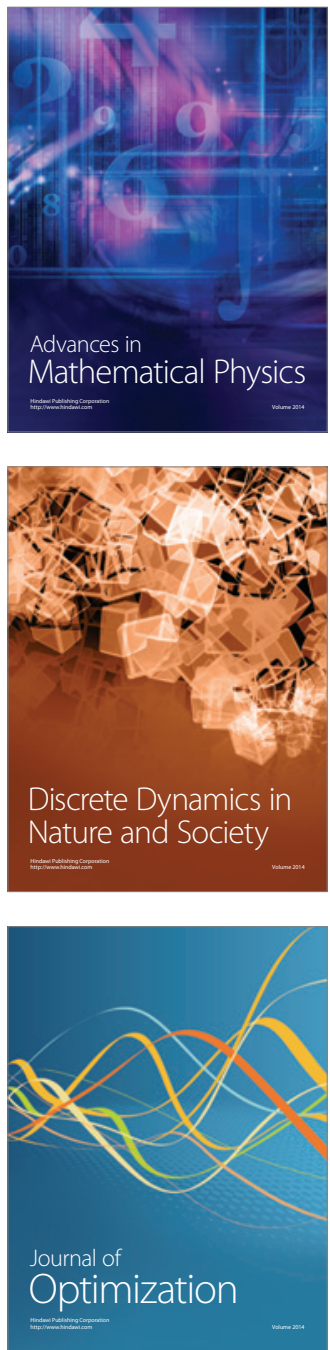